H A R VAR D

\title{
Farsighted Stability for Roommate Markets
}

\author{
Bettina Klaus \\ Flip Klijn \\ Markus Walzl
}

\section{Working Paper}

09-135 


\title{
Farsighted Stability for Roommate Markets*
}

\author{
Bettina Klaus $^{\dagger} \quad$ Flip Klijn ${ }^{\ddagger} \quad$ Markus Walzl ${ }^{\S}$
}

May 2009

\begin{abstract}
Using a bi-choice graph technique (Klaus and Klijn, 2009), we show that a matching for a roommate market indirectly dominates another matching if and only if no blocking pair of the former is matched in the latter (Proposition 1). Using this characterization of indirect dominance, we investigate von Neumann-Morgenstern farsightedly stable sets. We show that a singleton is von Neumann-Morgenstern farsightedly stable if and only if the matching is stable (Theorem 1). We also present roommate markets with no and with a non-singleton von Neumann-Morgenstern farsightedly stable set (Examples 1 and 2).

JEL classification: C62, C71, C78.
\end{abstract}

Keywords: core, farsighted stability, one- and two-sided matching, roommate markets, von Neumann-Morgenstern stability.

${ }^{*}$ B. Klaus thanks the Netherlands Organisation for Scientific Research (NWO) for its support under grant VIDI-452-06-013. F. Klijn's research was supported through the Spanish Plan Nacional $I+D+I$ (SEJ2005-01690 and ECO2008-04784), the Generalitat de Catalunya (SGR2005-00626 and the Barcelona GSE Research Network), and the Consolider-Ingenio 2010 (CSD2006-00016) program.

${ }^{\dagger}$ Corresponding author: Harvard Business School, Baker Library|Bloomberg Center 437, Soldier Field, Boston, MA 02163, USA; e-mail: bklaus@hbs.edu

†Institute for Economic Analysis (CSIC), Campus UAB, 08193 Bellaterra (Barcelona), Spain; e-mail: flip.klijn@iae.csic.es

${ }^{\S}$ Department of Economics, Bamberg University, Feldkirchenstr. 21, 96045 Bamberg, Germany; e-mail: markus.walzl@uni-bamberg. de 


\section{Introduction}

In a roommate market (Gale and Shapley, 1962), a finite set of agents has to be partitioned into pairs (roommates) and singletons. We refer to such a partition as a matching. Each agent has strict preferences over each of the other agents (i.e., sharing a room with him/her) and staying alone (or relying on an outside option). Hence, a roommate market is a simple example of hedonic coalition as well as network formation. In hedonic coalition formation (Bogomolnaia and Jackson, 2002), a set of agents has to be partitioned and agents have preferences over coalitions (i.e., all subsets of agents). Thus, for roommate markets coalition formation is restricted to coalitions of at most two agents. In network formation (Jackson and Watts, 2002), links between agents can be established and agents have preferences over their links (or even the entire network structure). Thus, for roommate markets network formation is restricted to at most one link per agent (and agents have preferences over this direct link only). Moreover, a roommate market can be interpreted as an extension of one of the most famous and simplest types of (two-sided) matching markets, a so-called marriage market. In a marriage market, agents are either male or female, and a man (woman) only wants to be matched to a woman (man) or to him(her)self. This setting is equivalent to a roommate market where the set of agents consists of two disjoint subsets and every agent in a certain subset prefers staying alone to being matched to another agent in the same subset. Hence, roommate markets are a particularly interesting class of matching markets because they lie in the "intersection" of network and coalition formation models.

In all the matching, coalition, and network models mentioned above, stability is a central property. For roommate markets, a matching is stable if all roommates are mutually acceptable and no pair of agents would prefer to be roommates instead of having their current matches. For marriage and roommate markets this (pairwise) stability notion is known to be equivalent to core stability. However, when extending the class of marriage markets to the class of roommate markets a problem occurs: while the core for a marriage market is always non-empty, the core of a roommate market can well be empty (Gale and Shapley, 1962). As a consequence, roommate markets can be considered an important benchmark for the development of solution concepts for matching, network, and coalition formation models that may exhibit an empty core or an empty set of stable matchings or network/coalition structures. ${ }^{1}$

Solution concepts can be either categorized as myopic or farsighted. The core would be an example of a myopic solution concept based on a direct dominance relation that formalizes the existence of blocking pairs. Using this direct dominance relation, Ehlers (2007) and Wako (2008) analyze von Neumann-Morgenstern stable sets for marriage markets. $^{2}$ By adding enforceability (loosely speaking, the way a matching changes into an-

\footnotetext{
${ }^{1}$ For more general models of matching as well as coalition or network formation, various stability notions exist and pairwise stability is no longer equivalent to core stability.

${ }^{2}$ Ehlers (2007) gives a necessary and sufficient condition for a set of matchings to be a von Neumann-
} 
other matching when blocking pairs are matched) to direct dominance, myopic dynamic solution concepts can be considered as well. In one branch of the "dynamic literature," individual decisions to match with other agents (or to establish a link or join a coalition) are myopic because agents only consider the new enforced matching (or network or coalition) and ignore the fact that the new matching itself could be un-stable as well. Myopic blocking dynamics of this kind have been introduced by Roth and Vande Vate (1990) for marriage markets and have been analyzed for couples markets by Klaus and Klijn (2007) and for (solvable) roommate markets by Diamantoudi et al. (2004). A model of dynamic network formation based on myopic blocking has been proposed by Jackson and Watts (2002) and modified to roommate markets by Klaus et al. (2008).

In contrast to the myopic approach, another branch of the literature models individuals as farsighted, i.e., agents do not only consider their new match but also potential future changes. Based on the concept of indirect dominance proposed by Harsanyi (1974) and formalized by Chwe (1994), several contributions investigate farsighted decision making and stability in abstract social situations (e.g., Greenberg, 1990; Chwe, 1994; Xue, 1998), hedonic coalition formation (e.g., Diamantoudi and Xue, 2003), or network formation (e.g., Page et al., 2005; Herings et al., 2008; Page and Wooders, 2008). The various solution concepts considered range from Greenberg's (1990) conservative stable standard of behavior as represented by the largest consistent set (Chwe, 1994) to the optimistic stable standard of behavior as represented by the von Neumann-Morgenstern farsightedly stable sets. Recently, Mauleon et al. (2008) analyzed von Neumann-Morgenstern farsightedly stable sets for two-sided matching markets. According to Mauleon et al. (2008), for marriage markets and many-to-one matching markets with responsive preferences, the only von Neumann-Morgenstern farsightedly stable sets are the singletons that consist of the stable matchings.

Here, we are interested in von Neumann-Morgenstern farsightedly stable sets for roommate markets. First, we use the bi-choice graph technique of Klaus and Klijn (2009) to give a simple characterization of indirect dominance (Proposition 1). Then, we show that a singleton matching is a von Neumann-Morgenstern farsightedly stable set if and only if the matching is stable (Theorem 1). Moreover, a pair of matchings can never be a von Neumann-Morgenstern farsightedly stable set (Lemma 4). However, we provide examples of roommate markets that exhibit no von Neumann-Morgenstern farsightedly stable set (Example 1) and a non-singleton von Neumann-Morgenstern farsightedly stable set (Example 2), respectively.

The paper is organized as follows. In Section 2 we present the roommate market model. In Section 3, we introduce von Neumann-Morgenstern farsightedly stable sets. Section 4 contains the results and Section 5 concludes.

Morgenstern stable set in a marriage market. Wako (2008) showed that each marriage market has a unique von Neumann-Morgenstern stable set. Other myopic solution concepts for roommate markets include maximum stable matchings (Tan, 1990), stable partitions (Tan, 1991), almost stable matchings (Abraham et al., 2006), p-stable matchings (Iñarra et al., 2008a), and absorbing sets (Iñarra et al., $2008 b)$. 


\section{The Model}

In a roommate market, a finite set of agents $N$ has to be partitioned into pairs (roommates) and singletons. Each agent $i \in N$ has preferences $R_{i}$ over sharing a room with any of the agents in $N \backslash\{i\}$ and having a room for himself (or consuming an outside option such as living off-campus). Agents' preferences are total orders ${ }^{3}$ over $N$. In particular, for all $i \in N, k R_{i} j$ and $j R_{i} k$ if and only if $j=k$. The strict preference and indifference relation associated with $R_{i}$ are denoted by $P_{i}$ and $I_{i}$, respectively. If $i P_{i} j$ then $j$ is unacceptable to $i$. Since the set of agents $N$ remains fixed throughout this paper, we simply denote a roommate market (Gale and Shapley, 1962) by its preference profile $R=\left(R_{i}\right)_{i \in N}$.

A solution to a roommate market, a matching $\mu$, is a partition of $N$ into pairs and singletons. Alternatively, a matching is a function $\mu: N \rightarrow N$ of order two, i.e., for all $i \in N, \mu(\mu(i))=i$. We denote the set of matchings for all roommate markets defined for the set of agents $N$ by $\mathcal{M}$. Agent $\mu(i)$ is agent $i$ 's mate, i.e., the agent with whom he is matched to share a room (possibly himself). For $S \subseteq N, \mu(S)$ denotes the set of mates of agents in $S$, i.e., $\mu(S)=\{\mu(i): i \in S\}$. If $\mu(i)=i$ then we call $i$ a single. With some abuse of notation we write $\mu R_{i} \mu^{\prime}$ if and only if $\mu(i) R_{i} \mu^{\prime}(i)$.

A matching $\mu$ is blocked by a pair $\{i, j\} \subseteq N$ (possibly $i=j$ ) if $j P_{i} \mu(i)$ and $i P_{j} \mu(j)$. If $\{i, j\}$ blocks $\mu$, then $\{i, j\}$ is called a blocking pair for $\mu$. A pair $\{i, j\} \subseteq N$ (possibly $i=j$ ) that is not a blocking pair for $\mu$ is called a non-blocking pair for $\mu$.

A matching $\mu$ is blocked by a coalition $S \subseteq N$ if there exists a matching $\mu^{\prime}$ such that $\mu^{\prime}(S)=S$ and for all $i \in S, \mu^{\prime}(i) P_{i} \mu(i)$. If $S$ blocks $\mu$, then $S$ is called a blocking coalition for $\mu$.

Next, we introduce the enforceability notion that we will use throughout the paper, i.e., in the following we describe how a coalition of agents can enforce a matching $\mu^{\prime}$ starting from a matching $\mu$. For any matching $\mu$ and any coalition $S \subseteq N$ we say that $\mu^{\prime}$ results from $\mu$ by matching $S$ if $\mu^{\prime}(S)=S$ and for all $k \in N \backslash S$,

$$
\mu^{\prime}(k):= \begin{cases}k & \text { if } \mu(k) \in S, \\ \mu(k) & \text { if } \mu(k) \notin S ;\end{cases}
$$

i.e., coalition $S$ is (re)matched among itself, previous mates of agents in $S$ who are not in $S$ themselves become single, and all other agents have the same mates as before. We write this as $\mu \rightarrow_{S} \mu^{\prime}$. If for some pair $\{i, j\}, \mu \rightarrow_{\{i, j\}} \mu^{\prime}$, then we assume, without loss of generality, that $\mu^{\prime}(i)=j \cdot{ }^{4}$ In this case we say that the (possibly non-blocking) pair $\{i, j\}$ is satisfied.

\footnotetext{
${ }^{3} \mathrm{~A}$ total order is a binary relation that satisfies antisymmetry, transitivity, and totality (comparability).

${ }^{4}$ The only other possibility is that $i \neq j, \mu^{\prime}(i)=i$, and $\mu^{\prime}(j)=j$. In that case $\mu^{\prime}$ also results from $\mu$ by matching $\{i, i\}$ or $\{j, j\}$.
} 
Note that if a coalition $S \subseteq N$ blocks a matching $\mu$, then there exists a pair $\{i, j\} \subseteq S$ (possibly $i=j$ ) that blocks $\mu$. Furthermore, $\mu \rightarrow_{S} \mu^{\prime}$ implies that there exist disjoint pairs $\left\{i_{1}, j_{1}\right\}, \ldots,\left\{i_{L-1}, j_{L-1}\right\}$ (possibly for some $l \in\{1, \ldots, L-1\}, i_{l}=j_{l}$ ) such that $\mu=\mu_{1} \rightarrow\left\{i_{1}, j_{1}\right\} \mu_{2} \rightarrow_{\left\{i_{2}, j_{2}\right\}} \cdots \rightarrow_{\left\{i_{L-1}, j_{L-1}\right\}} \mu_{L}=\mu^{\prime}$.

A matching is individually rational if there is no blocking pair $\{i, j\}$ with $i=j$. We denote the set of individually rational matchings for roommate market $R$ by $I(R)$. A matching is stable if there is no blocking pair. We denote the set of stable matchings for roommate market $R$ by $S(R)$. A roommate market $R$ is solvable if $S(R) \neq \emptyset$. Otherwise it is called unsolvable. Note that for any roommate market the set of stable matchings equals the core (due to the fact that the existence of any blocking coalition induces the existence of a blocking pair as already mentioned before).

A marriage market (Gale and Shapley, 1962) is a roommate market such that $N$ is the union of two disjoint sets $M$ and $W$ (men and women), and each agent in $M$ (respectively $W$ ) prefers being alone to being matched with any other agent in $M$ (respectively $W$ ). An individually rational matching for a marriage market respects the partition of agents into two types and never matches two men or two women.

Gale and Shapley (1962) showed that all marriage markets are solvable and provided an unsolvable roommate market (Gale and Shapley, 1962, Example 3).

\section{Von Neumann-Morgenstern Farsighted Stability}

Harsanyi (1974) criticizes stability notions based on myopic decision making; he argues that coalitions may enforce a myopically not very attractive outcome in order to set a chain of events in motion that in the end will lead to a preferred outcome for the coalition. The following indirect dominance notion incorporates this insight.

Matching $\mu^{\prime}$ indirectly dominates matching $\mu$, denoted by $\mu^{\prime} \gg \mu$, if there exists a sequence of matchings $\mu=\mu_{1}, \ldots, \mu_{L}=\mu^{\prime}$ and a sequence of pairs $\left\{i_{1}, j_{1}\right\}, \ldots,\left\{i_{L-1}, j_{L-1}\right\}$ such that for all $l \in\{1, \ldots, L-1\}, \mu_{l} \rightarrow\left\{i_{l}, j_{l}\right\} \mu_{l+1}$ and for all $k \in\left\{i_{l}, j_{l}\right\}, \mu^{\prime} P_{k} \mu_{l} .{ }^{5}$ We refer to such a sequence of pairs as an indirect dominance path of pairs (from $\mu$ to $\mu^{\prime}$ ) and to the resulting sequence of matchings $\mu=\mu_{1}, \ldots, \mu_{L}=\mu^{\prime}$ as an indirect dominance path of matchings (from $\mu$ to $\mu^{\prime}$ ).

Note that using coalitions instead of pairs would not change this definition as long as $\mu$ is individually rational (since any change by a coalition can be obtained by a sequence of disjoint pairs in this case). ${ }^{6}$ Moreover, if coalitions are used only minor changes in the

\footnotetext{
${ }^{5}$ Hence, we follow the standard myopic blocking dynamics used in various papers (e.g., Roth and Vande Vate, 1990; Diamantoudi et al., 2004; Klaus et al., 2008) by assuming that in the sequence of matchings from $\mu$ to $\mu^{\prime}$, any matching $\mu_{l+1}$ results from $\mu_{l}$ by matching a pair.

${ }^{6}$ To see that there is a matching $\mu^{\prime}$ that indirectly dominates an individually irrational matching $\mu$ by an indirect dominance path of coalitions but does not indirectly dominate $\mu$ by an indirect dominance path of pairs, consider the following example. Let $N=\{1,2,3,4\}$ and $1 P_{1} 2 P_{1} 4 P_{1} 3,1 P_{2} 3 P_{2} 2 P_{2} 4$,
} 
proofs are needed to show that all our results still hold.

A set of matchings $V \subseteq \mathcal{M}$ is farsightedly internally stable if for all $\mu, \mu^{\prime} \in V, \mu^{\prime} \ngtr \mu$. Every set of matchings $V$ with cardinality $|V|=1$ is farsightedly internally stable.

A set of matchings $V \subseteq \mathcal{M}$ is farsightedly externally stable if for all matchings $\mu \notin V$ there exists a matching $\mu^{\prime} \in V$ such that $\mu^{\prime} \gg \mu$. The set of all matchings $\mathcal{M}$ is farsightedly externally stable.

A set of matchings $V \subseteq \mathcal{M}$ is a von Neumann-Morgenstern ( $v N M$ ) farsightedly stable set if it is farsightedly internally and externally stable. Von Neumann-Morgenstern farsightedly stable sets represent Greenberg's (1990) optimistic stable standard of behavior (OSSB) based on the indirect dominance relation.

\section{Results}

We first introduce the bi-choice graph technique of Klaus and Klijn (2009) to prove a useful characterization of indirect dominance. As a by-product, the bi-choice graph technique offers a simple construction of indirect dominance paths between individually rational matchings.

Let $R$ be a roommate market and $\mu, \mu^{\prime} \in I(R), \mu \neq \mu^{\prime}$, such that (a) there is no blocking pair $\left\{i^{\prime}, j^{\prime}\right\}$ for $\mu$ with $\mu^{\prime}\left(i^{\prime}\right)=j^{\prime}$ and (b) there is no blocking pair $\{i, j\}$ for $\mu^{\prime}$ with $\mu(i)=j$. We consider the following bi-choice graph $G\left(\mu, \mu^{\prime}\right)=(N, E)$. The set of vertices is the set of agents $N$. The set of edges $E$ consists of the following three types of edges. Let $i, j \in N$. Then,

$\boldsymbol{E} 1$. there is a continuous directed edge from $i$ to $j$, denoted by $i \bullet \longrightarrow j$ if $j=$ $\mu(i) P_{i} \mu^{\prime}(i)$, i.e., agent $i$ strictly prefers his match $j=\mu(i)$ under $\mu$ to his match under $\mu^{\prime}$;

E2. there is a discontinuous directed edge from $i$ to $j$, denoted by $i \bullet-\rightarrow \cdot j$ if $j=$ $\mu^{\prime}(i) P_{i} \mu(i)$, i.e., agent $i$ strictly prefers his match $j=\mu^{\prime}(i)$ under $\mu^{\prime}$ to his match under $\mu$;

E3. there is a (continuous) undirected edge between $i$ and $j$, denoted by $i \cdot-\boldsymbol{l} j$ if $j=\mu(i) I_{i} \mu^{\prime}(i)$, i.e., agent $i$ is indifferent between his match $j=\mu(i)$ under $\mu$ and his match under $\mu^{\prime}$. Note that for $j=i=\mu(i) I_{i} \mu^{\prime}(i)$ we allow for an undirected edge from $i$ to himself; we call such an edge a loop:

$3 P_{3} 4 P_{3} 2 P_{3} 1,3 P_{4} 1 P_{4} 4 P_{4} 2$. Then, $\mu=[\{1,4\},\{2,3\}] \rightarrow_{N} \mu^{\prime}=[\{1,2\},\{3,4\}]$ is an indirect dominance path of coalitions from the individually irrational matching $\mu$ to $\mu^{\prime}$. On any indirect dominance path of pairs from $\mu$ to $\mu^{\prime}$, however, agent 1 or agent 3 has to become single at some point. But both agents are matched in an individually irrational way at $\mu^{\prime}$ - a contradiction to farsighted stability using pairs. Hence, using coalitions in the definition of indirect dominance paths leads to strictly more indirect dominance relations than using pairs. 


\section{Lemma 1. Bi-choice graph components}

Let $R$ be a roommate market and $\mu, \mu^{\prime} \in I(R), \mu \neq \mu^{\prime}$, such that (a) there is no blocking pair $\left\{i^{\prime}, j^{\prime}\right\}$ for $\mu$ with $\mu^{\prime}\left(i^{\prime}\right)=j^{\prime}$ and (b) there is no blocking pair $\{i, j\}$ for $\mu^{\prime}$ with $\mu(i)=j$. Let $i \in N$. Then, agent $i$ 's component of $G\left(\mu, \mu^{\prime}\right)$ either

(a) equals $i \bullet \longrightarrow j$ for some agent $j$ (i.e., $i \circlearrowright$ if $j=i$ ), or

(b) is a directed even cycle, i.e., there is a directed path starting from $i$ that induces a closed cycle $c_{i}=\left(i_{1}, i_{2}, i_{3}, \ldots, i_{p}\right)$ consisting of an even number $p \geq 4$ of agents (with $i \in\left\{i_{1}, \ldots, i_{p}\right\}$ and $i_{r} \neq i_{s}$ for all $r \neq s$ ) where continuous and discontinuous edges alternate.

Klaus and Klijn (2009, Lemma 1) prove Lemma 1 for stable matchings, but explain why the result easily generalizes to Lemma 1 as stated above (Klaus and Klijn, 2009, Footnote 6).

Our first result characterizes the indirect dominance relation on the domain of individually rational matchings. Mauleon et al. (2008, Lemma 1) is a corresponding result for marriage markets.

\section{Proposition 1. A characterization of indirect dominance}

Let $R$ be a roommate market and $\mu, \mu^{\prime} \in I(R)$ with $\mu \neq \mu^{\prime}$. Then, $\mu^{\prime} \gg \mu$ if and only if there is no blocking pair $\{i, j\}$ for $\mu^{\prime}$ with $\mu(i)=j$.

Proof.

" $\Rightarrow$ " Suppose $\mu^{\prime} \gg \mu$ and there exists a blocking pair $\{i, j\}$ for $\mu^{\prime}$ with $\mu(i)=j$.

Let $\left\{i_{1}, j_{1}\right\}, \ldots,\left\{i_{L-1}, j_{L-1}\right\}$ be an indirect dominance path of pairs from $\mu$ to $\mu^{\prime}$. Let $\mu=\mu_{1}, \ldots, \mu_{L}=\mu^{\prime}$ be the corresponding indirect dominance path of matchings. Then, since $\mu(i)=j$ and $\mu^{\prime}(i) \neq j$, there is a smallest index $l^{*} \in\{1, \ldots, L-1\}$ that labels the first time one of the two agents in $\{i, j\}$ actively participates in the indirect dominance path, i.e., $l^{*} \in\{1, \ldots, L-1\}$ is the smallest index such that $\{i, j\} \cap\left\{i_{l^{*}}, j_{l^{*}}\right\} \neq \emptyset$. Let $k \in\{i, j\} \cap\left\{i_{l^{*}}, j_{l^{*}}\right\}$. By the definition of an indirect dominance path, $\mu^{\prime}(k) P_{k} \mu_{l^{*}}(k)$. By the minimality of $l^{*}, \mu_{l^{*}}(k)=\mu(k)$. Thus, $\mu^{\prime}(k) P_{k} \mu(k)$, in contradiction to $\{i, j\}$ with $\mu(i)=j$ and $k \in\{i, j\}$ being a blocking pair for $\mu^{\prime}$. Hence, $\mu^{\prime} \gg \mu$ implies that there is no blocking pair $\{i, j\}$ for $\mu^{\prime}$ with $\mu(i)=j$.

$\underline{\prime \prime} \Leftarrow$ " Assume that there is no blocking pair $\{i, j\}$ for $\mu^{\prime}$ with $\mu(i)=j$. We now explicitly construct an indirect dominance path of pairs from $\mu$ to $\mu^{\prime}$ in order to show $\mu^{\prime} \gg \mu$.

First, take any blocking pair $\{i, j\}$ for $\mu$ that is matched under $\mu^{\prime}$ (if any) and match agents $i$ and $j$. Now take any blocking pair for the resulting matching that is matched under $\mu^{\prime}$ (if any) and match the involved agents. Continue satisfying blocking pairs one by one in this way, until we obtain a matching $\mu^{*}$ such that there is no blocking pair for $\mu^{*}$ that is matched under $\mu^{\prime}$. Note that since $\mu \in I(R)$, all blocking pairs that are satisfied in this procedure are of cardinality 2 and $\mu^{*} \in I(R)$. 
Note that once a blocking pair is satisfied in the procedure it will never be unmatched in the remainder of the procedure. So,

$$
\mu^{*} \gg \mu \text {. }
$$

Hence, if $\mu^{*}=\mu^{\prime}$ then we are done. Suppose $\mu^{*} \neq \mu^{\prime}$. Then, $\mu^{\prime}, \mu^{*} \in I(R), \mu^{\prime} \neq \mu^{*}$, and by construction of $\mu^{*}$, (a) there is no blocking pair $\left\{i^{\prime}, j^{\prime}\right\}$ for $\mu^{*}$ with $\mu^{\prime}\left(i^{\prime}\right)=j^{\prime}$.

Suppose there is a blocking pair $\left\{i^{*}, j^{*}\right\}$ for $\mu^{\prime}$ with $\mu^{*}\left(i^{*}\right)=j^{*}$. Since (by assumption) there is no blocking pair $\{i, j\}$ for $\mu^{\prime}$ with $\mu(i)=j, \mu\left(i^{*}\right) \neq j^{*}$. Since $\mu^{*}\left(i^{*}\right)=j^{*}$ and by the construction of $\mu^{*}$, then $\left\{i^{*}, j^{*}\right\}$ is a blocking pair for $\mu$ that is matched under $\mu^{\prime}$; contradicting that $\left\{i^{*}, j^{*}\right\}$ is a blocking pair for $\mu^{\prime}$. Therefore, (b) there is no blocking pair $\left\{i^{*}, j^{*}\right\}$ for $\mu^{\prime}$ with $\mu^{*}\left(i^{*}\right)=j^{*}$.

So, Lemma 1 applies to the bi-choice graph $G\left(\mu^{\prime}, \mu^{*}\right)$. Without loss of generality, assume that there is exactly one directed even cycle $\left(i_{1}, i_{2}, i_{3}, \ldots, i_{p}\right)$ in $G\left(\mu^{\prime}, \mu^{*}\right)$ (if not, then repeat the following argument for each directed even cycle). ${ }^{7}$ This implies that matchings $\mu^{\prime}$ and $\mu^{*}$ only differ in the way the agents in $\left\{i_{1}, i_{2}, i_{3}, \ldots, i_{p}\right\}$ are matched. Assume, without loss of generality, that $\mu^{\prime}\left(i_{1}\right)=i_{2}$, i.e., there is a continuous directed edge from $i_{1}$ to $i_{2}$. Then, all agents $i_{k}$ with $k$ being odd, prefer their mates at $\mu^{\prime}$ to their mates at $\mu^{*}$ and all agents $i_{l}$ with $l$ being even, prefer their mates at $\mu^{*}$ to their mates at $\mu^{\prime}$.

Let $\tilde{\mu}_{1}:=\mu^{*}$ and $\tilde{\mu}_{1} \rightarrow\left\{i_{1}\right\} \tilde{\mu}_{2}$ (agent $i_{1}$ un-matches from $\left.\mu^{*}\left(i_{1}\right)=i_{p}\right)$. Then, at the new matching $\tilde{\mu}_{2}$ agent $i_{p}$ is single and prefers to be matched with $\mu^{\prime}\left(i_{p}\right)=i_{p-1}$. Thus, since $p-1$ is odd, $\left\{i_{p-1}, i_{p}\right\}$ is a blocking pair for $\tilde{\mu}_{2}$. Let $\tilde{\mu}_{2} \rightarrow_{\left\{i_{p-1}, i_{p}\right\}} \tilde{\mu}_{3}$. Then, at $\tilde{\mu}_{3}$ agent $i_{p-2}$ is single and prefers to be matched with $\mu^{\prime}\left(i_{p-2}\right)=i_{p-3}$. Then, since $p-3$ is odd, $\left\{i_{p-3}, i_{p-2}\right\}$ is a blocking pair for $\tilde{\mu}_{3}$. Let $\tilde{\mu}_{3} \rightarrow\left\{i_{p-3}, i_{p-2}\right\} \tilde{\mu}_{4}$, etc.

Since $p$ is even, we cover the complete cycle without overlaps and obtain a sequence of matchings $\mu^{*}=\tilde{\mu}_{1}, \ldots, \tilde{\mu}_{\frac{p}{2}+2}=\mu^{\prime}$ such that for all $l \in\left\{2, \ldots, \frac{p}{2}+1\right\}, \tilde{\mu}_{l+1}$ results from $\tilde{\mu}_{l}$ by matching pair $\left\{i_{p-2 l+3}, i_{p-2 l+4}\right\}$ and for all $k \in\left\{i_{p-2 l+3}, i_{p-2 l+4}\right\}, \mu^{\prime} P_{k} \tilde{\mu}_{l}$. Also note that for $l=1, \mu^{\prime} P_{i_{1}} \tilde{\mu_{1}}=\tilde{\mu}_{l}$. Hence,

$$
\mu^{\prime} \gg \mu^{*} .
$$

Recall that in the construction of $\mu^{*}$ out of $\mu$ once a blocking pair is satisfied it will never be unmatched until we obtain $\mu^{*}$. In fact, for any such blocking pair $\{i, j\}$ and for any $k \in\{i, j\}, \mu^{\prime}(k)=\mu^{*}(k)$. But then (1) and (2) imply $\mu^{\prime} \gg \mu$, which completes the proof.

By the following lemma, only individually rational matchings can be part of any vNM farsightedly stable set.

\section{Lemma 2. Individual rationality of $v N M$ farsightedly stable sets}

Let $V$ be a $v N M$ farsightedly stable set and $\mu \in V$. Then, $\mu$ is individually rational.

\footnotetext{
${ }^{7}$ There is at least one such cycle because otherwise $\mu^{\prime}=\mu^{*}$; a contradiction.
} 
Proof. Suppose $\mu \in V$ is not individually rational. Then, there exists a blocking pair $\{i, i\}$ for $\mu$. Consider $\mu \rightarrow_{\{i\}} \mu^{\prime}$. By farsighted internal stability, $\mu^{\prime} \notin V$. By farsighted external stability, there exists a $\mu^{\prime \prime} \in V$ such that $\mu^{\prime \prime} \gg \mu^{\prime}$. Suppose $\mu^{\prime \prime}=\mu$. Then, $\mu \gg \mu^{\prime}$. By the proof of $" \Rightarrow$ " of Proposition 1, there is no blocking pair for $\mu$ matched under $\mu^{\prime}$, which is in contradiction to $\mu \rightarrow\{i\} \mu^{\prime}$. Hence, $\mu^{\prime \prime} \neq \mu$.

Let $\left\{i_{1}, j_{1}\right\}, \ldots,\left\{i_{L-1}, j_{L-1}\right\}$ be an indirect dominance path of pairs from $\mu^{\prime}$ to $\mu^{\prime \prime}$. Since $\mu^{\prime}(i)=i$, either $\mu^{\prime \prime}(i)=i$ or $\mu^{\prime \prime}(i) P_{i} \mu^{\prime}(i)$. In either case, $\{i\},\left\{i_{1}, j_{1}\right\}, \ldots,\left\{i_{L-1}, j_{L-1}\right\}$ is an indirect dominance path of pairs from $\mu$ to $\mu^{\prime \prime}$. Hence, $\mu^{\prime \prime} \gg \mu$, which is in contradiction to farsighted internal stability.

The next theorem extends Theorem 1 in Mauleon et al. (2008) to roommate markets.

\section{Theorem 1. Stable matchings and $v N M$ farsightedly stable sets}

A singleton $\{\mu\}$ is a vNM farsightedly stable set if and only if $\mu$ is stable.

Proof.

" $\Rightarrow$ " Suppose $\{\mu\}$ is a vNM farsightedly stable set and $\mu$ is not stable. Then, there is a blocking pair $\{i, j\}$ for $\mu$. By Lemma 2, $\mu \in I(R)$. Consider $\mu \rightarrow_{\{i, j\}} \mu^{\prime}$. Note that $\mu^{\prime} \in I(R)$. By Proposition $1, \mu \gg \mu^{\prime}$ because there exists a blocking pair $\{i, j\}$ for $\mu$ with $\mu^{\prime}(i)=j$. This contradicts farsighted external stability of $\{\mu\}$.

$\underline{\prime \prime} \Leftarrow$ " Let $\mu$ be stable. Since any singleton is farsighted internally stable, we only have to prove that $\{\mu\}$ is farsighted externally stable. Let $\mu^{\prime} \neq \mu$ be a (possibly individually irrational) matching. Let $\left\{i_{1}^{\prime}, \ldots, i_{k}^{\prime}\right\}$ be the agents that under $\mu^{\prime}$ are matched in an individually irrational way. Consider $\mu^{\prime}$ and unmatch all agents in $\left\{i_{1}^{\prime}, \ldots, i_{k}^{\prime}\right\}$. Denote the resulting matching by $\mu^{\prime \prime}$.

Since $\mu$ is stable and $\mu^{\prime \prime} \in I(R)$, Proposition 1 immediately implies $\mu \gg \mu^{\prime \prime}$. Let $\left\{i_{1}, j_{1}\right\}, \ldots,\left\{i_{L-1}, j_{L-1}\right\}$ be an indirect dominance path of pairs from $\mu^{\prime \prime}$ to $\mu$. Since $\mu$ is individually rational, all agents in $\left\{i_{1}^{\prime}, \ldots, i_{k}^{\prime}\right\}$ will strictly prefer $\mu$ to $\mu^{\prime}$. This implies that $\left\{i_{1}^{\prime}\right\}, \ldots,\left\{i_{k}^{\prime}\right\},\left\{i_{1}, j_{1}\right\}, \ldots,\left\{i_{L-1}, j_{L-1}\right\}$ is an indirect dominance path of pairs from $\mu^{\prime}$ to $\mu$. Hence, $\mu \gg \mu^{\prime}$.

The " $\Leftarrow$ "-part is related to Diamantoudi and Xue (2003) who show that for hedonic games with strict preferences any partition that is in the core indirectly dominates any other partition.

While Theorem 1 implies the existence of a vNM farsightedly stable set in any solvable roommate market (and in particular in any marriage market), the following example presents an (unsolvable) roommate market without any vNM farsightedly stable set.

\section{Example 1. A roommate market without a vNM farsightedly stable set}

We consider the following unsolvable roommate market with three agents $N=\{1,2,3\}$. Table 1 lists agents' preferences in its columns, e.g., agent 1's preferences are such that $2 P_{1} 3 P_{1} 1$. 


\begin{tabular}{|c|c|c|}
\hline agent 1 & agent 2 & agent 3 \\
\hline \hline 2 & 3 & 1 \\
3 & 1 & 2 \\
1 & 2 & 3 \\
\hline
\end{tabular}

Table 1: Example 1 - preferences

The set of matchings equals $\mathcal{M}=I(R)=\left\{\mu_{0}, \mu_{1}, \mu_{2}, \mu_{3}\right\}$ where

$$
\begin{aligned}
& \mu_{0}=[\{1\},\{2\},\{3\}], \\
& \mu_{1}=[\{1,2\},\{3\}], \\
& \mu_{2}=[\{1,3\},\{2\}], \\
& \mu_{3}=[\{1\},\{2,3\}] .
\end{aligned}
$$

Using Proposition 1 it is readily verified that the only indirect dominance relations between the matchings are the following: $\mu_{1} \gg \mu_{2} \gg \mu_{3} \gg \mu_{1}, \mu_{1} \gg \mu_{0}, \mu_{2} \gg \mu_{0}$, and $\mu_{3} \gg \mu_{0}$. Suppose there exists a vNM farsightedly stable set $V$. Clearly, by farsighted internal stability, $|V|<2$. Thus, $V=\{\mu\}$ for some $\mu \in \mathcal{M}$. Since the set of stable matchings $S(R)=\emptyset$, this contradicts Theorem 1 .

The next results give some more insights into the structure of von NeumannMorgenstern farsightedly stable sets.

\section{Lemma 3. Mutual blocking in vNM farsightedly stable sets}

Let $V$ be a vNM farsightedly stable set. For any two matchings $\mu_{1}, \mu_{2} \in V$ with $\mu_{1} \neq \mu_{2}$ there is a blocking pair $\left\{i_{1}, j_{1}\right\}$ for $\mu_{1}$ with $\mu_{2}\left(i_{1}\right)=j_{1}$ and there is a blocking pair $\left\{i_{2}, j_{2}\right\}$ for $\mu_{2}$ with $\mu_{1}\left(i_{2}\right)=j_{2}$.

Proof. Let $\mu_{1}, \mu_{2} \in V$ with $\mu_{1} \neq \mu_{2}$. By Lemma $2, \mu_{1}, \mu_{2} \in I(R)$. Suppose that there is no blocking pair $\left\{i_{1}, j_{1}\right\}$ for $\mu_{1}$ with $\mu_{2}\left(i_{1}\right)=j_{1}$. Then, by Proposition $1, \mu_{1} \gg \mu_{2}$; contradicting farsighted internal stability. Hence, there is a blocking pair $\left\{i_{1}, j_{1}\right\}$ for $\mu_{1}$ with $\mu_{2}\left(i_{1}\right)=j_{1}$. The proof that there is a blocking pair $\left\{i_{2}, j_{2}\right\}$ for $\mu_{2}$ with $\mu_{1}\left(i_{2}\right)=j_{2}$ is similar.

Next, we prove that no vNM farsightedly stable set can be composed of exactly two elements.

Lemma 4. Two element vNM farsightedly stable sets do not exist For any $v N M$ farsightedly stable set $V,|V| \neq 2$.

Proof. Suppose $V=\left\{\mu_{1}, \mu_{2}\right\}$ with $\mu_{1} \neq \mu_{2}$. Then, by Lemma 3, there is a blocking pair $\left\{i_{1}, j_{1}\right\}$ for $\mu_{1}$ with $\mu_{2}\left(i_{1}\right)=j_{1}$ and there is a blocking pair $\left\{i_{2}, j_{2}\right\}$ for $\mu_{2}$ with $\mu_{1}\left(i_{2}\right)=j_{2}$. 
Take any blocking pair $\{i, j\}$ for $\mu_{2}$ that is matched under $\mu_{1}$ (note that there is at least one such blocking pair) and match agents $i$ and $j$. Now take any blocking pair for the resulting matching that is matched under $\mu_{1}$ (if any) and match the involved agents. Continue satisfying blocking pairs one by one in this way, until we obtain a matching $\mu_{2}^{\prime}$ such that there is no blocking pair for $\mu_{2}^{\prime}$ that is matched under $\mu_{1}$. By Lemma $2, \mu_{1}, \mu_{2} \in I(R)$. Hence, all blocking pairs that are satisfied in this procedure are of cardinality 2 and $\mu_{2}^{\prime} \in I(R)$. By construction and Proposition $1, \mu_{2} \ngtr \mu_{2}^{\prime}$. Furthermore, by construction, there are no blocking pairs $\left\{i^{\prime}, j^{\prime}\right\}$ for $\mu_{2}^{\prime}$ with $\mu_{2}\left(i^{\prime}\right)=j^{\prime}$. Hence, by Proposition 1, $\mu_{2}^{\prime} \gg \mu_{2}$. Thus, by farsighted internal stability, $\mu_{2}^{\prime} \notin V$.

Next, by farsighted internal stability we have that $\mu_{1} \gg \mu_{2}$. Thus, by Proposition 1 , there exists a blocking pair $\{i, j\}$ for $\mu_{1}$ with $\mu_{2}(i)=j$. Note that neither $i$ nor $j$ are then involved in a blocking pair of $\mu_{2}$ that is matched under $\mu_{1}$. Hence, by construction of $\mu_{2}^{\prime}, \mu_{2}^{\prime}(i)=j$. Thus, there exists a blocking pair $\{i, j\}$ for $\mu_{1}$ with $\mu_{2}^{\prime}(i)=j$ and by Proposition $1, \mu_{1} \gg \mu_{2}^{\prime}$. Since also $\mu_{2} \gg \mu_{2}^{\prime}$, this is in contradiction to farsighted external stability of $V$.

Lemma 4 excludes the existence of a vNM farsightedly stable set with two matchings for roommate markets. A stronger result for the subclass of marriage markets is stated in Mauleon et al. (2008, Theorem 2), according to which any vNM farsightedly stable set is a singleton that consists of a stable matching. Independently from Mauleon et al. (2008) we have established the nonexistence of vNM farsightedly stable sets with three matchings for marriage markets. We have relegated the statement and proof of this result to the Appendix (Lemma 5).

Our final example shows, however, that Lemma 5 cannot be generalized to the class of roommate markets. It presents an (unsolvable) roommate market with a vNM farsightedly stable set that consists of three matchings. In particular, the example shows that roommate markets can exhibit a vNM farsightedly stable set with un-stable matchings, which is not the case for marriage markets according to Mauleon et al. (2008, Theorem 2).

\section{Example 2. A three element vNM farsightedly stable set}

We consider an unsolvable roommate market with six agents $N=\{1, \ldots, 6\}$. Table 2 lists agents' preferences in its columns, e.g., agent 1 's preferences are such that $2 P_{1} 3 P_{1} 1 \cdots$ where "..." represents any ordering of the remaining agents. 


\begin{tabular}{|c|c|c|c|c|c|}
\hline agent 1 & agent 2 & agent 3 & agent 4 & agent 5 & agent 6 \\
\hline \hline 2 & 3 & 1 & 6 & 4 & 5 \\
3 & 1 & 2 & 5 & 6 & 4 \\
1 & 2 & 3 & 4 & 5 & 6 \\
$\vdots$ & $\vdots$ & $\vdots$ & $\vdots$ & $\vdots$ & $\vdots$ \\
\hline
\end{tabular}

Table 2: Example 2 - preferences

Define

$$
\begin{aligned}
& \mu_{0}=[\{1\}, \ldots,\{6\}], \\
& \mu_{1}=[\{1,2\},\{3\},\{4,5\},\{6\}], \\
& \mu_{2}=[\{2,3\},\{1\},\{5,6\},\{4\}], \\
& \mu_{3}=[\{1,3\},\{2\},\{4,6\},\{5\}], \\
& \mu_{4}=[\{1,2\},\{3\},\{5,6\},\{4\}], \\
& \mu_{5}=[\{1,2\},\{3\},\{4,6\},\{5\}], \\
& \mu_{6}=[\{2,3\},\{1\},\{4,6\},\{5\}], \\
& \mu_{7}=[\{2,3\},\{1\},\{4,5\},\{6\}], \\
& \mu_{8}=[\{1,3\},\{2\},\{4,5\},\{6\}], \\
& \mu_{9}=[\{1,3\},\{2\},\{5,6\},\{4\}] .
\end{aligned}
$$

Let $V=\left\{\mu_{1}, \mu_{2}, \mu_{3}\right\}$. To see that $V$ is internally stable, consider w.l.o.g. $\mu_{1}$. First, notice that $\{4,5\}$ is a blocking pair for $\mu_{2}$ that is matched under $\mu_{1}$. So, by Proposition 1 , $\mu_{2}$ does not indirectly dominate $\mu_{1}$. Second, notice that $\{1,2\}$ is a blocking pair for $\mu_{3}$ that is matched under $\mu_{1}$. So, again by Proposition $1, \mu_{3}$ does not indirectly dominate $\mu_{1}$. Hence, $\mu_{1}$ is not indirectly dominated by another matching in $V$. Analogously it can be shown that $\mu_{2}$ and $\mu_{3}$ are not indirectly dominated by another matching in $V$. Hence, $V$ is internally stable.

To see that $V$ is externally stable, consider a matching $\mu \notin V$ (possibly individually irrational) and construct the following sequence of matchings.

Step I: Unmatch pairs of agents that are matched in an individually irrational way and denote the resulting matching by $\tilde{\mu}$. If $\tilde{\mu} \in V$, we have established an indirect dominance path from $\mu$ to a matching in $V$.

Step II: Suppose that $\tilde{\mu} \notin V$. Note that $\tilde{\mu} \in I(R)$.

Suppose $\tilde{\mu}$ has no matched pairs. Then, $\tilde{\mu}=\mu_{0}$. Then, Step $I$ and subsequently matching $\{1,2\}$ and $\{4,5\}$ constitutes an indirect dominance path from $\mu$ to $\mu_{1}$. Hence, there exists an indirect dominance path from $\mu$ to a matching in $V$. 
Suppose $\tilde{\mu}$ has exactly one matched pair. By the symmetric construction of the example, assume without loss of generality that $\tilde{\mu}=[\{1,2\},\{3\},\{4\},\{5\},\{6\}]$. Then, Step I and subsequently matching $\{4,5\}$ constitutes an indirect dominance path from $\mu$ to $\mu_{1}$. Hence, there exists an indirect dominance path from $\mu$ to a matching in $V$.

Suppose $\tilde{\mu}$ has exactly two matched pairs. Then, for some $i \in\{4, \ldots, 9\}, \tilde{\mu}=\mu_{i}$. Let $\tilde{\mu}=\mu_{4}=[\{1,2\},\{3\},\{5,6\},\{4\}]$. Then, Step $I$ and subsequently matching $\{2,3\}$ constitutes an indirect dominance path from $\mu$ to $\mu_{2} \in V$. Let $\tilde{\mu}=\mu_{5}=[\{1,2\},\{3\},\{4,6\},\{5\}]$. Then, Step $I$ and subsequently matching $\{2,3$,$\} and \{5,6\}$ constitutes an indirect dominance path from $\mu$ to $\mu_{2} \in V$. Similarly, if for some $i=6,7,8,9, \tilde{\mu}=\mu_{i}$, then there exists an indirect dominance path from $\mu$ to a matching in $V$. We list one indirect dominance path by means of blocking pairs for each matching $\mu_{i}, i \in\{4, \ldots, 9\}$, in Table 3 . Hence, any $\mu \notin V$ is indirectly dominated by a matching in $V$ and $V$ is externally stable. $\diamond$

\begin{tabular}{|c|c|c|}
\hline matching outside of $V$ & blocking pairs to be matched & resulting matching in $V$ \\
\hline \hline$\mu_{4}$ & $\{2,3\}$ & $\mu_{2}$ \\
$\mu_{5}$ & $\{2,3\},\{5,6\}$ & $\mu_{2}$ \\
$\mu_{6}$ & $\{1,3\}$ & $\mu_{3}$ \\
$\mu_{7}$ & $\{1,3\},\{4,6\}$ & $\mu_{3}$ \\
$\mu_{8}$ & $\{1,2\}$ & $\mu_{1}$ \\
$\mu_{9}$ & $\{1,2\},\{4,5\}$ & $\mu_{1}$ \\
\hline
\end{tabular}

Table 3: Example 2 - blocking pairs on an indirect dominance path into $V$

\section{Conclusion}

In this paper, we have shown a strong relation between stable matchings of a roommate market, i.e., matchings that are not myopically blocked by a pair of agents, and singleton vNM farsightedly stable sets: a singleton set is a vNM farsightedly stable set if and only if its element is stable (Theorem 1). Thus, a matching is myopically stable if and only if it farsightedly (indirectly) dominates any other matching. Hence, for roommate markets, the myopic notion of (pairwise) stability also induces farsighted stability. For the subclass of marriage markets, according to Mauleon et al. (2008, Theorems 2), also the converse is true: the only vNM farsightedly stable sets are singleton sets with stable matchings.

We find that results for roommate markets can differ in two fundamental ways from those for marriage markets. First, for roommate markets it is possible that a vNM farsightedly stable set exists while no "myopic prediction" can be made (Example 2 describes a roommate market with no stable matchings, but with a non-empty vNM farsightedly stable set). Second, while the existence of stable matchings for marriage markets also 
guarantees a "farsighted prediction," for roommate markets it is possible that no vNM farsightedly stable set exists (Example 1). Hence, for an unsolvable roommate market there never exists a singleton vNM farsightedly stable set while a non-singleton vNM farsightedly stable set may or may not exist. For solvable roommate markets, at least one singleton vNM farsightedly stable set always exists and it is easy to construct solvable roommate markets (e.g., marriage markets) without non-singleton vNM farsightedly stable sets. Whether there exists a solvable roommate market with a non-singleton vNM farsightedly stable set, however, is currently an open question.

\section{A Appendix}

Lemma 5. Three element $v N M$ farsightedly stable sets do not exist for marriage markets For any vNM farsightedly stable set $V$ of a marriage market, $|V| \neq 3$.

Proof. Let $V$ be a non-singleton vNM farsightedly stable set and consider a graph with the set of vertices $V$ and directed edges $\sim_{\{i, j\}}$ where $\mu_{1} \leadsto_{\{i, j\}} \mu_{2}$ indicates that $\{i, j\}$ is a blocking pair for $\mu_{1}$ that is matched under $\mu_{2}$. Note that the graph has no loops, i.e., there is no edge from a matching $\mu_{1} \in V$ to itself. By Lemma 3, there exists at least one edge from any matching in $V$ to any other matching in $V$.

Let $\mu_{1}, \mu_{2} \in V, \mu_{1} \neq \mu_{2}$. Consider any $\{i, j\}$ with $\mu_{1} \leadsto\{i, j\} \mu_{2}$. Let $\mu_{1} \rightarrow_{\{i, j\}} \tilde{\mu}_{1}$, i.e., $\tilde{\mu}_{1}$ is the matching that results from $\mu_{1}$ by matching $\{i, j\}$. By internal stability of $V, \tilde{\mu}_{1} \notin V$. By external stability of $V$, there exists a matching $\mu_{1}^{\prime} \in V$ with $\mu_{1}^{\prime} \gg \tilde{\mu}_{1}$. By Lemma 2, $\mu_{1} \in I(R)$. So, by definition of $\tilde{\mu}_{1}$ and $\{i, j\}, \tilde{\mu}_{1} \in I(R)$. Note also that $\mu_{1}^{\prime} \in I(R)$. Hence, by Proposition 1, there is no blocking pair for $\mu_{1}^{\prime}$ that is matched under $\tilde{\mu}_{1}$. In particular, $\mu_{1}^{\prime} \neq \mu_{1}$. By Lemma 3 , however, there is a blocking pair $\left\{i_{1}^{\prime}, j_{1}^{\prime}\right\}$ for $\mu_{1}^{\prime}$ that is matched under $\mu_{1}$. Since $\mu_{1} \rightarrow_{\{i, j\}} \tilde{\mu}_{1}$, the only agents who have different mates under $\mu_{1}$ and $\tilde{\mu}_{1}$ are $i, j, \mu_{1}(i)$, and $\mu_{1}(j)$. Then, the only two possibilities of blocking pairs for $\mu_{1}^{\prime}$ that are matched under $\mu_{1}$ but not matched under $\tilde{\mu}_{1}$ are $\left\{i, \mu_{1}(i)\right\}$ and $\left\{j, \mu_{1}(j)\right\}$. Let w.l.o.g. $\left\{i, \mu_{1}(i)\right\}$ be a blocking pair for $\mu_{1}^{\prime}$ that is matched under $\mu_{1}$ but not matched under $\tilde{\mu}_{1}$. Hence, $\mu_{1}^{\prime} \sim_{\left\{i, \mu_{1}(i)\right\}} \mu_{1} \sim_{\{i, j\}} \mu_{2}$.

Suppose $V=\left\{\mu_{1}, \mu_{2}, \mu_{3}\right\}$. As there are no loops, $\mu_{1}^{\prime} \neq \mu_{1}$. Suppose that $\mu_{1}^{\prime}=\mu_{2}$. Then, $\mu_{2}=\mu_{1}^{\prime} \leadsto\left\{i, \mu_{1}(i)\right\} \mu_{1}$ implies that $i$ strictly prefers $\mu_{1}(i)$ to $\mu_{2}(i)=j$. However, since $\mu_{1} \leadsto\{i, j\} \mu_{2}$, agent $i$ strictly prefers $j$ to $\mu_{1}(i)$. This contradiction, together with $\mu_{1}^{\prime} \in V$, shows that $\mu_{1}^{\prime}=\mu_{3}$.

Let $\mu_{3} \rightarrow\left\{i, \mu_{1}(i)\right\} \tilde{\mu}_{3}$. By internal stability of $V, \tilde{\mu}_{3} \notin V$. By external stability of $V$, there exists a matching $\mu_{3}^{\prime} \in V$ with $\mu_{3}^{\prime} \gg \tilde{\mu}_{3}$. By Lemma $2, \mu_{3} \in I(R)$. So, by definition of $\tilde{\mu}_{3}$ and $\left\{i, \mu_{1}(i)\right\}, \tilde{\mu}_{3} \in I(R)$. Note also that $\mu_{3}^{\prime} \in I(R)$. Hence, by Proposition 1 , there is no blocking pair for $\mu_{3}^{\prime}$ that is matched under $\tilde{\mu}_{3}$. In particular, $\mu_{3}^{\prime} \neq \mu_{3}$. By Lemma 3 , however, there is a blocking pair $\left\{i_{3}^{\prime}, j_{3}^{\prime}\right\}$ for $\mu_{3}^{\prime}$ that is matched under $\mu_{3}$. Since $\mu_{3} \rightarrow\left\{i, \mu_{1}(i)\right\} \tilde{\mu}_{3}$, the only agents who have different mates under $\mu_{3}$ and $\tilde{\mu}_{3}$ are $i, \mu_{1}(i)$, $\mu_{3}(i)$, and $\mu_{3}\left(\mu_{1}(i)\right)$. The only two possibilities of blocking pairs for $\mu_{3}^{\prime}$ that are matched 
under $\mu_{3}$ but not matched under $\tilde{\mu}_{3}$ are $\left\{i, \mu_{3}(i)\right\}$ and $\left\{\mu_{1}(i), \mu_{3}\left(\mu_{1}(i)\right)\right\}$. So, $\mu_{3}^{\prime} \sim_{\left\{i_{3}^{\prime}, j_{3}^{\prime}\right\}} \mu_{3}$ for $\left(i_{3}^{\prime}, j_{3}^{\prime}\right)=\left(i, \mu_{3}(i)\right)$ or $\left(i_{3}^{\prime}, j_{3}^{\prime}\right)=\left(\mu_{3}\left(\mu_{1}(i)\right), \mu_{1}(i)\right)$.

As there are no loops, $\mu_{3}^{\prime} \neq \mu_{3}$. Suppose that $\mu_{3}^{\prime}=\mu_{1}$. Then, $\mu_{1}=\mu_{3}^{\prime} \hookrightarrow_{\left\{i_{3}^{\prime}, j_{3}^{\prime}\right\}}$ $\mu_{3}$ implies that $i_{3}^{\prime}$ strictly prefers $\mu_{3}$ to $\mu_{1}$. However, since $\mu_{3} \sim\left\{i, \mu_{1}(i)\right\} \mu_{1}$, agent $i_{3}^{\prime}$ strictly prefers $\mu_{1}$ to $\mu_{3}$. This contradiction, together with $\mu_{3}^{\prime} \in V$, shows that $\mu_{3}^{\prime}=\mu_{2}$. Summarizing, $\mu_{2} \hookrightarrow_{\left\{i_{3}^{\prime}, j_{3}^{\prime}\right\}} \mu_{3} \leadsto\left\{i, \mu_{1}(i)\right\} \mu_{1} \hookrightarrow_{\{i, j\}} \mu_{2}$.

Suppose $\left(i_{3}^{\prime}, j_{3}^{\prime}\right)=\left(i, \mu_{3}(i)\right)$. Then, $\mu_{2} P_{i} \mu_{1} P_{i} \mu_{3} P_{i} \mu_{2}$, which contradicts the transitivity of $P_{i}$. Hence, assume that $\left(i_{3}^{\prime}, j_{3}^{\prime}\right)=\left(\mu_{3}\left(\mu_{1}(i)\right), \mu_{1}(i)\right)$. If $\mu_{3}\left(\mu_{1}(i)\right)=i$, then again $\mu_{2} P_{i} \mu_{1} P_{i} \mu_{3} P_{i} \mu_{2}$. So, $i_{3}^{\prime}=\mu_{3}\left(\mu_{1}(i)\right) \neq i$. Moreover, $j_{3}^{\prime}=\mu_{1}(i) \neq \mu_{2}(i)=j$. Note that by Lemma 2 , all matchings in $V$ are individually rational. Hence, $\left\{i, \mu_{1}(i)\right\},\{i, j\}$, and $\left\{i_{3}^{\prime}, j_{3}^{\prime}\right\}$ are blocking pairs of cardinality 2. Suppose w.l.o.g. that agent $i$ is a man, i.e., $i \in M$. Then, agents $j$ and $\mu_{1}(i)$ are women, i.e., $j, \mu_{1}(i) \in W$. Since $j_{3}^{\prime}=\mu_{1}(i) \in W$, $\mu_{3}\left(\mu_{1}(i)\right)=i_{3}^{\prime} \in M$. Hence, $i$ and $i_{3}^{\prime}$ belong to another market side than $j$ and $j_{3}^{\prime}$. Since $i \neq i_{3}^{\prime}$ and $j \neq j_{3}^{\prime},\{i, j\} \cap\left\{i_{3}^{\prime}, j_{3}^{\prime}\right\}=\emptyset .^{8}$

Let $\{k, l\}$ be such that $\mu_{3} \leadsto\{k, l\} \mu_{2}$. Since $\mu_{3} \in I(R),\{k, l\}$ is a blocking pair of cardinality 2. Suppose w.l.o.g. that $k \in M$. Then, $i, k \in M$ and $j, l \in W$.

Case I: Suppose $\{k, l\} \cap\{i, j\} \neq \emptyset$. Hence, since $\mu_{2}(k)=l$ and $\mu_{2}(i)=j, k=i$ and $l=j$. Thus, $\{i, j\}$ is a blocking pair for $\mu_{1}$ and $\mu_{3}$ that is matched under $\mu_{2}$. Let $\mu_{2} \rightarrow_{\left\{i_{3}^{\prime}, j_{3}^{\prime}\right\}} \tilde{\mu}_{2}$. By internal stability of $V, \tilde{\mu}_{2} \notin V$. Note that all matchings in $V$ have a blocking pair that is matched under $\tilde{\mu}_{2}\left(\{i, j\}\right.$ or $\left.\left\{i_{3}^{\prime}, j_{3}^{\prime}\right\}\right)$. Hence, by $\tilde{\mu}_{2} \in I(R)$ and Proposition 1 , there exists no $\mu \in V$ with $\mu \gg \tilde{\mu}_{2}$, which is in contradiction to the assumption that $V=\left\{\mu_{1}, \mu_{2}, \mu_{3}\right\}$ is a vNM farsightedly stable set. Hence, $\{k, l\} \cap\{i, j\}=\emptyset$.

Case II: Suppose $k=i_{3}^{\prime}$. Then, $\mu_{2} \sim_{\left\{k, j_{3}^{\prime}\right\}} \mu_{3} \sim_{\{k, l\}} \mu_{2}$. Hence, $\mu_{2} P_{k} \mu_{3} P_{k} \mu_{2}$, which contradicts the transitivity of $P_{k}$.

Case III: Suppose $l=j_{3}^{\prime}$. Then, $\mu_{2} \sim\left\{i_{3}^{\prime}, l\right\} \mu_{3} \leadsto\{k, l\} \mu_{2}$. Hence, $\mu_{2} P_{l} \mu_{3} P_{l} \mu_{2}$, which contradicts the transitivity of $P_{l}$.

Case $I V$ : Since $i_{3}^{\prime}, k \in M$ and $j_{3}^{\prime}, l \in W$, this remaining case covers $\left\{i_{3}^{\prime}, j_{3}^{\prime}\right\} \cap\{k, l\}=\emptyset$. Let $\mu_{2} \rightarrow\left\{i_{3}^{\prime}, j_{3}^{\prime}\right\} \tilde{\mu}_{2}$. By internal stability of $V, \tilde{\mu}_{2} \notin V$. Note that all matchings in $V$ have a blocking pair that is matched under $\tilde{\mu}_{2}\left(\{i, j\},\{k, l\}\right.$, or $\left.\left\{i_{3}^{\prime}, j_{3}^{\prime}\right\}\right)$. Hence, by $\tilde{\mu}_{2} \in I(R)$ and Proposition 1, there exists no $\mu \in V$ with $\mu \gg \tilde{\mu}_{2}$, which is in contradiction to the assumption that $V=\left\{\mu_{1}, \mu_{2}, \mu_{3}\right\}$ is a vNM farsightedly stable set.

\footnotetext{
${ }^{8}$ This does not necessarily hold for a roommate market since in that case $i=j_{3}^{\prime}$ or $j=i_{3}^{\prime}$ cannot be excluded.
} 


\section{References}

Abraham, D. J., Biró, P., and Manlove, D. F. (2006): "Almost Stable" Matchings in the Roommates Problem." In T. Erlebach and G. Persiano, editors, Proceedings of WAOA 2005, Lecture Notes in Computer Science Vol. 3879, pages 1-14. Springer, Berlin/Heidelberg.

Bogomolnaia, A. and Jackson, M. (2002): "The Stability of Hedonic Coalition Structures." Games and Economic Behavior, 38: 201-230.

Chwe, M. S.-Y. (1994): "Farsighted Coalitional Stability." Journal of Economic Theory, 63: 299-325.

Diamantoudi, E., Miyagawa, E., and Xue, L. (2004): "Random Paths to Stability in the Roommate Problem." Games and Economic Behavior, 48: 18-28.

Diamantoudi, E. and Xue, L. (2003): "Farsighted Stability in Hedonic Games." Social Choice and Welfare, 21: 39-61.

Ehlers, L. (2007): "Von Neumann-Morgenstern Stable Sets in Matchings Problems." Journal of Economic Theory, 134: 537-547.

Gale, D. and Shapley, L. S. (1962): "College Admissions and the Stability of Marriage." American Mathematical Monthly, 69: 9-15.

Greenberg, J. (1990): The Theory of Social Situations: An Alternative Game-Theoretic Approach. Cambridge University Press, Cambridge.

Harsanyi, J. C. (1974): "An Equilibrium-Point Interpretation of Stable Sets and a Proposed Alternative Definition." Management Science, 20: 1472-1495.

Herings, J. J., Mauleon, A., and Vannetelbosch, V. (2008): "Farsightedly Stable Networks." Games and Economic Behavior, forthcoming.

Iñarra, E., Larrea, C., and Molis, E. (2008a): "Random Paths to P-stability in the Roommate Problem." International Journal of Game Theory, 36: 461-471.

Iñarra, E., Larrea, C., and Molis, E. (2008b): "The Stability of the Roommate Problem Revisited." Mimeo (May).

Jackson, M. O. and Watts, A. (2002): "The Evolution of Social and Economic Networks." Journal of Economic Theory, 106: 265-295.

Klaus, B. and Klijn, F. (2007): "Paths to Stability for Matching Markets with Couples." Games and Economic Behavior, 58: 154-171. 
Klaus, B. and Klijn, F. (2009): "Smith and Rawls Share a Room: Stability and Medians." Harvard Business School Working Paper 09-111 (http://www.hbs.edu/research/pdf/09-111.pdf).

Klaus, B., Klijn, F., and Walzl, M. (2008): "Stochastic Stability for Roommate Markets." Meteor Research Memorandum 08/010 (http://ideas.repec.org/p/dgr/umamet/2008010.html).

Mauleon, A., Vannetelbosch, V., and Vergote, W. (2008): "Von Neumann-Morgenstern Farsightedly Stable Sets in Two-Sided Matching." Core Discussion Paper 2008/16 (revised version: December 2008).

Page, F. H. J. and Wooders, M. H. (2008): "Strategic Basins of Attraction, the Path Dominance Core, and Network Formation Games." Games and Economic Behavior, forthcoming.

Page, F. H. J., Wooders, M. H., and Kamat, S. (2005): "Networks and Farsighted Stability." Journal of Economic Theory, 120: 257-269.

Roth, A. E. and Vande Vate, J. (1990): "Random Paths to Stability in Two-Sided Matching." Econometrica, 58: 1475-1480.

Tan, J. J. M. (1990): "A Maximum Stable Matching for the Roommates Problem." BIT, 29: $631-640$.

Tan, J. J. M. (1991): "A Necessary and Sufficient Condition for the Existence of a Complete Stable Matching." Journal of Algorithms, 12: 154-178.

Wako, J. (2008): "A Note on Existence and Uniqueness of vNM Stable Sets in Marriage Games." Mimeo (July).

Xue, L. (1998): "Coalitional Stability under Perfect Foresight." Economic Theory, 11: 603-627. 